\title{
Thermal Denaturation, Renaturation, and Aggregation of a Double-Helical Polysaccharide Xanthan in Aqueous Solution
}

\author{
By Yasuhiro MATSUDA, ${ }^{\dagger}$ Yusuke BIYAJIMA, and Takahiro SATO*
}

\begin{abstract}
Xanthan samples prepared in different denaturation and renaturation conditions were studied by circular dichroism (CD) and size exclusion chromatography equipped with a multi-angle light scattering on-line detector (SEC-MALS). It turned out from $\mathrm{CD}$ that xanthan looses the double helical conformation in pure water at $80^{\circ} \mathrm{C}$ and almost recovers locally the native conformation in the renaturation. On the other hand, SEC-MALS results indicated that the xanthan double helix dissociates into two single chains in the denaturation concentration $c_{\mathrm{d}} \leq 1 \mathrm{mg} / \mathrm{cm}^{3}$, but not at $c_{\mathrm{d}}=10 \mathrm{mg} / \mathrm{cm}^{3}$ due to the increase in the ionic strength by xanthan itself, stabilizing the xanthan dimer. Furthermore the xanthan single chain dissociated at $c_{\mathrm{d}} \leq 1 \mathrm{mg} / \mathrm{cm}^{3}$ reconstructs the intramolecular double helical structure with a hairpin loop in the renaturation, while the xanthan dimer denatured at $c_{\mathrm{d}}=10 \mathrm{mg} / \mathrm{cm}^{3}$ aggregates by mismatched pairing. The degree of aggregation is enhanced at higher added salt concentration at the renaturation.

KEY WORDS: Double Helix / Polysaccharide / Denaturation / Renaturation / Aggregation / Size Exclusion Chromatography / MultiAngle Light Scattering /
\end{abstract}

A microbial polysaccharide, xanthan, is used as additives to foods, detergents, cosmetics, etc. to control their rheological properties. Its main chain is the same as cellulose and every other glucose residue bears a charged trisaccharide side chain. It was reported that the native xanthan takes a double-helical structure and exhibits an order-disorder transition in aqueous solution by changing temperature and ionic strength. ${ }^{1}$ The disordered or denatured xanthan recovers its native circular dichroism (CD) by quenching and adding salt to the solution, and in what follows we call this phenomenon as the renaturation of xanthan. However, the viscosity of the renatured xanthan solution does not recover the native one, ${ }^{2}$ so that the local conformation of renatured xanthan is similar to that of the native but its global conformation is different from that of native xanthan. When the concentration of xanthan is high enough, the renatured xanthan solution even forms a gel, which is another important property of xanthan in industrial applications. $^{3}$

Studies on the denaturation and renaturation of xanthan in aqueous solution, being basically important to control its solution properties, were made previously by several workers. Liu et $a l .{ }^{4-6}$ investigated the denaturation of the xanthan double helix in $0.01 \mathrm{M}$ aqueous $\mathrm{NaCl}$ at $25-80^{\circ} \mathrm{C}$ by static light scattering, viscosity, and optical rotation. Kawakami et al. ${ }^{7}$ made sedimentation equilibrium, viscosity, and optical rotation experiments on denatured xanthan after quenching at $25^{\circ} \mathrm{C}$ and adding $\mathrm{NaCl}$ of 0.1 or $0.01 \mathrm{M}$. They found that the xanthan dimer gradually dissociates into single chains with increasing heating time (longer than $7 \mathrm{~h}$ ) in the denaturation, and also indicated that denatured xanthan forms aggregates after quench at finite concentrations.
On the other hand, Oviatt and Brant ${ }^{2}$ and also Capron et al. ${ }^{8}$ denatured xanthan in $0.1 \mathrm{M}$ aqueous $\mathrm{NaCl}$ upon heating above $110^{\circ} \mathrm{C}$ and renatured it by quenching at $25^{\circ} \mathrm{C}$. The latter research group studied the renatured xanthan mainly by lowangle light scattering and viscometry, and concluded that the xanthan dimer dissociates into two single chains above $110^{\circ} \mathrm{C}$ and the renatured xanthan unimer takes the double helical structure with a hairpin loop at the renaturation concentration as low as $1 \mathrm{mg} / \mathrm{cm}^{3}$. On the other hand, Oviatt and Brant ${ }^{2}$ investigated xanthan renatured at higher concentration (10$30 \mathrm{mg} / \mathrm{cm}^{3}$ ) by viscoelastic measurements, and found viscosity enhancement of xanthan solutions after renaturation, which indicates the formation of large aggregates of xanthan chains. However, they did not study the global conformation of the renatured xanthan aggregates.

The previous studies indicate that the denaturation, renaturation, and aggregation of xanthan in aqueous solution are complex phenomena depending on various experimental conditions, and systematic studies are necessary to fully understand these phenomena. In the present study, we have undertaken the size exclusion chromatography with a multi-angle light scattering on-line detector (SEC-MALS) on xanthan samples prepared in different denaturation and renaturation conditions. From the molar mass distribution of the xanthan samples obtained by SEC-MALS, we have found that the denaturation of xanthan in pure water at $80^{\circ} \mathrm{C}$ sensitively depends on the xanthan concentration $c_{\mathrm{d}}$ in the denaturation. Furthermore, the molar mass dependence of the radius of gyration for renatured xanthan samples, obtained also by SEC-MALS, gave us the information about conformations of xanthan prepared in different denaturation and renaturation conditions.

Department of Macromolecular Science, Osaka University, 1-1 Machikaneyama-cho, Toyonaka 560-0043, Japan

Present Address: Department of Materials Science and Chemical Engineering, Faculty of Engineering, Shizuoka University, 3-5-1 Johoku, Naka-ku, Hamamatsu, 432-8561, Japan

*To whom correspondence should be addressed (Tel/Fax: +81-6-6850-5461, E-mail: tsato@ chem.sci.osaka-u.ac.jp). 
Table I. Molecular characteristics of xanthan samples used

\begin{tabular}{ccc}
\hline Sample & $M_{\mathrm{w}} / 10^{5}$ & $M_{\mathrm{w}} / M_{\mathrm{n}}$ \\
\hline S14 & 14 & 1.6 \\
S5 & 5.1 & 1.1 \\
S1 & 1.2 & $<1.1$ \\
\hline
\end{tabular}

Table II. Denaturation and renaturation conditions of aqueous xanthan solutions examined

\begin{tabular}{ccccc}
\hline Solution \# & $c_{\mathrm{d}} / \mathrm{mg} \mathrm{cm}^{-3}$ & Heating time & $c_{\mathrm{r}} / \mathrm{mg} \mathrm{cm}^{-3}$ & $C_{\mathrm{s}} / \mathrm{M}$ \\
\hline 1 & 0.10 & $2 \mathrm{~h}$ & 0.095 & 0.01 \\
2 & 1.0 & $2 \mathrm{~h}$ & 0.50 & 0.01 \\
3 & 1.0 & $2 \mathrm{~h}$ & 0.50 & 0.1 \\
4 & 10 & $30 \mathrm{~min}$ & 5.0 & 0.01 \\
5 & 10 & $30 \mathrm{~min}$ & 5.0 & 0.1 \\
\hline
\end{tabular}

\section{EXPERIMENTAL}

\section{Xanthan Samples and Test Solutions}

Three stock xanthan samples (S14, S5, and S1) were used in this study. They were sonicated, purified, and fractionated by repeated fractional precipitation; samples S5 and S1 were further fractionated by liquid crystal fractionation in an established procedure. ${ }^{9,10}$ Weight-average molecular weights $M_{\mathrm{w}}$ and ratios of $M_{\mathrm{w}}$ to the number-average molecular weight $M_{\mathrm{n}}$ ) of the samples, obtained by SEC-MALS (see below) are listed in Table I.

Each xanthan sample was first dissolved into pure water to prepare a solution with the mass concentration of xanthan $c_{\mathrm{d}}$ $\left(=0.1,1.0\right.$, or $\left.10 \mathrm{mg} / \mathrm{cm}^{3}\right)$. This solution was thermally denatured at $80^{\circ} \mathrm{C}$ for $30 \mathrm{~min}-2 \mathrm{~h}$, quenched at $25^{\circ} \mathrm{C}$ for $30 \mathrm{~min}$, and diluted with aqueous $\mathrm{NaCl}$ to make the polymer concentration $c_{\mathrm{r}}$. The final salt concentration $C_{\mathrm{s}}$ was adjusted to 0.01 or $0.1 \mathrm{M}$. If $c_{\mathrm{r}}$ was high enough, the viscosity of the renatured solution kept increasing after the addition of $\mathrm{NaCl}$, but it stopped increasing by dilution of the solution with aqueous $\mathrm{NaCl}$ of the same $C_{\mathrm{s}}$. The Huggins plot for the diluted renatured solution exhibited the normal concentration dependence, indicating that the state of renatured xanthan did not change by dilution.

On the basis of the above viscometric observation, each quenched solution was left for $24 \mathrm{~h}$ after the addition of $\mathrm{NaCl}$, and diluted with aqueous $\mathrm{NaCl}$ of the same $C_{\mathrm{s}}$ to a suitable concentration $c$ for the following measurements. Conditions for preparing renatured solutions are summarized in Table II.

\section{SEC-MALS Measurements}

SEC-MALS measurements were made for aqueous solutions of the thermally treated xanthan mentioned above, as well as of the native, using a GPC-900 system of JASCO Corporation with a column SB-806M HQ of Shodex Corporation, a DAWN DSP MALS system of Wyatt Corporation, and an RI-930 refractive index (RI) detector. The MALS system is equipped with $\mathrm{He}-\mathrm{Ne}$ laser of the wavelength $\lambda$ of $632.8 \mathrm{~nm}$. As the eluent, 0.01 or $0.1 \mathrm{M}$ aqueous solution of $\mathrm{NaCl}$ was used, the flow rate was $0.5 \mathrm{~mL} / \mathrm{min}$, and the temperature was controlled at $40{ }^{\circ}$ C. Sample solutions were filtered with DISMIC-25cs (cellulose acetate, $0.80 \mu \mathrm{m}$ ) of Toyo Roshi Co. Ltd. before injection to SEC.

The excess scattering intensity $\Delta I_{\theta}$ at the scattering angle $\theta$ and the excess refractive index $\Delta n$ of the solution over those of the solvent were measured as functions of the elution volume $V_{\mathrm{e}}$. They were converted to the excess Rayleigh ratio $R_{\theta}$ and the mass concentration $c$ using the relations

$$
R_{\theta}=\Phi_{\theta} \Delta I_{\theta}, c=(\partial n / \partial c)^{-1} \Delta n
$$

where $\Phi_{\theta}$ is the instrument constant depending on $\theta$ and the refractive index of the eluent, and $\partial n / \partial c$ is the refractive index increment of the polymer solution. The instrument constant was determined using toluene as the standard material. Referring to literature data, $0.138 \mathrm{~cm}^{3} / \mathrm{g}^{4,9}$ was used for $\partial n / \partial c$ (at constant solvent chemical potential) of aqueous xanthan at both $C_{\mathrm{s}}=0.01$ and $0.1 \mathrm{M}$.

For dilute polymer solutions, $R_{\theta}$ is related to the molar mass $M$, the second virial coefficient $A_{2}$, and the intramolecular interference factor $P(k)$ by

$$
\frac{K c}{R_{\theta}}=\frac{1}{M P(k)}+2 A_{2} c
$$

where $K$ is the optical constant and $k$ is the magnitude of the scattering vector. The second virial term correction was made by using the expression

$$
A_{2}=b M^{-a}
$$

with previously reported values $b=5.1 \times 10^{-3} \mathrm{~cm}^{3} \mathrm{~mol} / \mathrm{g}^{2}$ and $a=0.185$ in $0.1 \mathrm{M}$ aqueous $\mathrm{NaCl}^{9,11}$ and $b=0.37$ $\mathrm{cm}^{3} \mathrm{~mol} / \mathrm{g}^{2}$ and $a=0.41$ in $0.01 \mathrm{M}$ aqueous $\mathrm{NaCl}$ for xanthan. ${ }^{4}$ These asymptotic power law dependences were demonstrated to hold in ranges of $M$ from $10^{5}$ to $10^{7}$ in $0.1 \mathrm{M}$ $\mathrm{NaCl}$ and from $10^{5}$ to $10^{6}$ in $0.01 \mathrm{M} \mathrm{NaCl}$, and are expected to hold at higher $M$.

In a low $k$ region, $P(k)$ can be expanded in the form $P(k)=1-\left(\left\langle S^{2}\right\rangle k\right)^{2} / 3+\cdots$, where $\left\langle S^{2}\right\rangle^{1 / 2}$ is the radius of gyration, but for polymers with large $\left\langle S^{2}\right\rangle^{1 / 2}$ higher order terms in this expansion become important. For the Gaussian chain, we can use the Debye function

$$
P_{\mathrm{D}}(k)=\left(2 / u^{2}\right)\left(e^{-u}+u-1\right)
$$

as the fitting function of $P(k)$, where $u \equiv\left\langle S^{2}\right\rangle^{1 / 2} k .^{12}$ On the other hand, Yoshizaki and Yamakawa ${ }^{13}$ calculated $P(k)$ for the wormlike chain. Their result can be approximated by $P_{\mathrm{D}}(k)$ at $u$ smaller than a critical value $u^{*}$. Here, we define $u^{*}$ as the maximum $u$ where $P_{\mathrm{D}}(k)$ agrees with $P(k)$ of Yoshizaki and Yamakawa within $2 \%$. Figure 1 shows $u^{*}$ as a function of the Kuhn statistical segment number $N$ calculated by $M / 2 q M_{\mathrm{L}}$ with the persistence length $q$ and the molar mass per unit contour length $M_{\mathrm{L}}$.

\section{Circular Dichroism}

Circular dichroism (CD) spectra were measured on native, denatured, and renatured xanthan with a JASCO J-720WO 


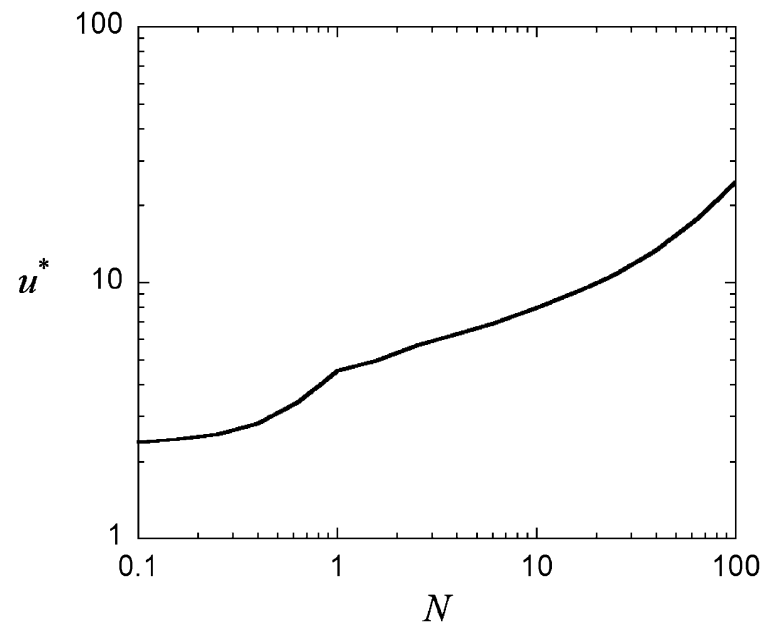

Figure 1. Maximum $u$ value where $P_{\mathrm{D}}(k)$ agrees with $P(k)$ for the wormlike chain within $2 \%$, as a function of the Kuhn segment number $N$.

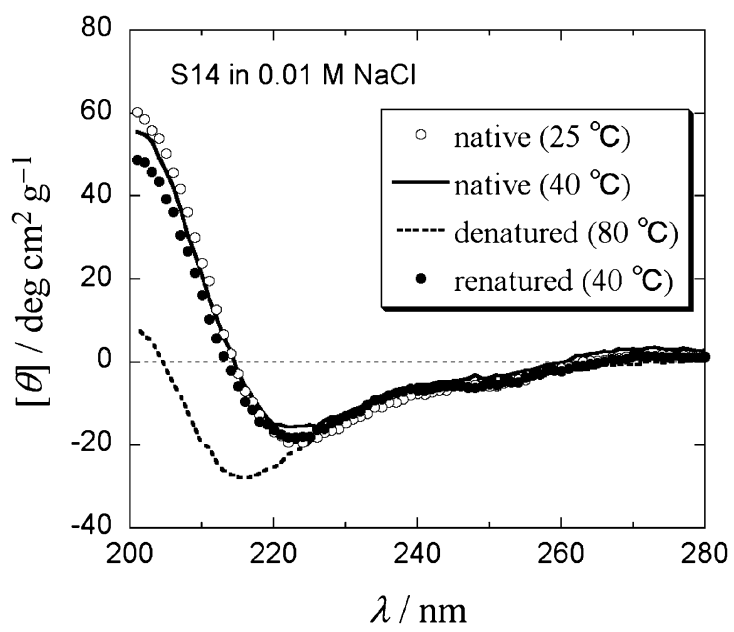

Figure 2. $C D$ spectra of native, thermally denatured, and renatured xanthan samples $\mathrm{S} 14$ in $0.01 \mathrm{M}$ aqueous $\mathrm{NaCl}\left(c=1 \mathrm{mg} / \mathrm{cm}^{3}\right)$.

spectropolarimeter. A quartz cell with $0.5 \mathrm{~cm}$ optical pass length and with a thermostat jacket was used for the measurements.

\section{RESULTS AND DISCUSSION}

\section{Circular Dichroism}

Figure 2 shows $\mathrm{CD}$ spectra of native and thermally denatured and renatured xanthan sample $\mathrm{S} 14$ in $0.01 \mathrm{M}$ aqueous $\mathrm{NaCl}\left(c=1 \mathrm{mg} / \mathrm{cm}^{3}\right)$. We observe a strong positive peak and a weaker negative peak at around 200 and $220 \mathrm{~nm}$, respectively, for the native at $25^{\circ} \mathrm{C}$ (unfilled circles) and $40{ }^{\circ} \mathrm{C}$ (solid curve). Morris et al. ${ }^{14}$ assigned these two peaks to carboxylate and carboxylate ester chromophores attaching to xanthan side chains. The CD spectra at the two temperatures are essentially identical, demonstrating that the helical conformation intrinsic to the native xanthan maintains at $40{ }^{\circ} \mathrm{C}$ (the temperature of SEC-MALS measurements).
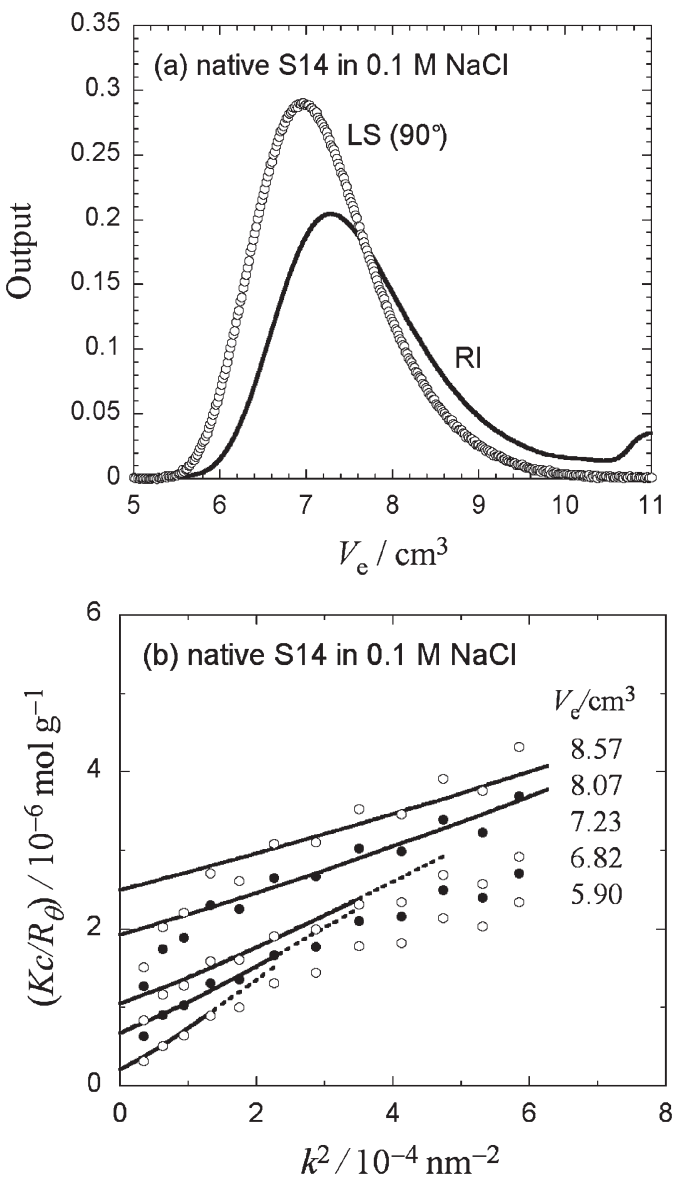

Figure 3. (a) SEC chromatograms for the native sample $\mathrm{S} 14$ by MALS $\left(\theta=90^{\circ}\right)$ and $\mathrm{RI}$ detectors; (b) plots of $K c / R_{\theta} v s . k^{2}$ for the native sample $\mathrm{S} 14$ at different $V_{\mathrm{e}}$.

While the positive peak much reduces for the denatured sample in pure water heated at $80^{\circ} \mathrm{C}$ (dashed curve), the $\mathrm{CD}$ spectrum is almost recovered to that of the native sample after quench to $40{ }^{\circ} \mathrm{C}$, indicating that the double helical structure of xanthan is locally reconstructed by the renaturation.

\section{Analyses of SEC-MALS Data}

Size exclusion chromatograms for the native sample S14 by MALS $\left(\theta=90^{\circ}\right)$ and RI detectors are shown in Figure 3a. The baseline of the chromatogram of the MALS detector agrees at both sides of the peak, but not for the chromatogram of the RI detector. The imperfectness (the tailing effect) of SEC may be due to loading not sufficiently dilute test solution, but accurate MALS measurements were impossible at lower concentrations c. We have made SEC-MALS measurements on xanthan solutions with $c$ as low as possible, and analyzed the chromatogram by using the baseline on the smaller $V_{\mathrm{e}}$ side, noting that such analyses might introduce some errors into $M$ and $\left\langle S^{2}\right\rangle^{1 / 2}$ at larger $V_{\mathrm{e}}$ (or lower $M$ ).

Figure $3 \mathrm{~b}$ shows plots of $K c / R_{\theta} v s . k^{2}$ for the native sample $\mathrm{S} 14$ at different $V_{\mathrm{e}}$ selected. In the first approximation, $M$ and $\left\langle S^{2}\right\rangle^{1 / 2}$ were determined at each $V_{\mathrm{e}}$ from $K c / R_{\theta}$ data over all the $k$ range measured, using eqs 2 and 3 with $P(k)=P_{\mathrm{D}}(k)$ 


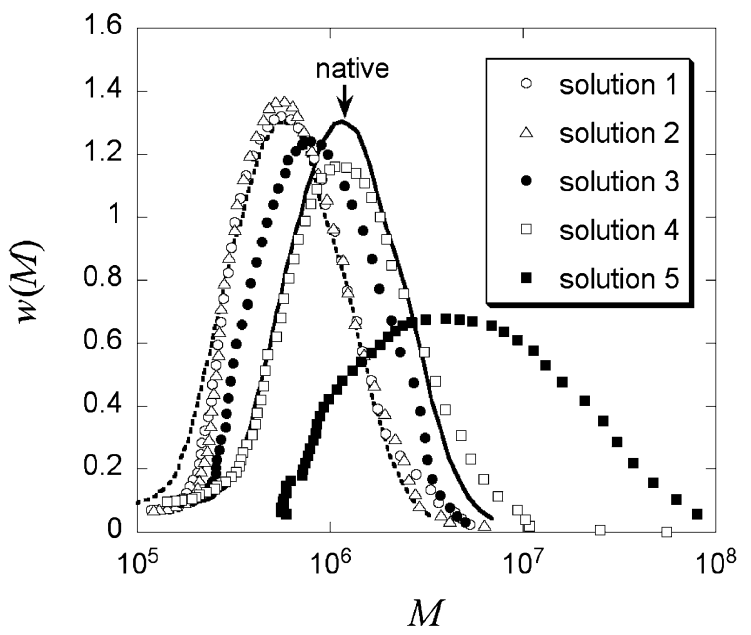

Figure 4. Molar mass distributions $w(M)$ of sample S14 renatured under the conditions specified in Table II.

given by eq 4 . Then, the Kuhn segment number $N$ was calculated by $M / 2 q M_{\mathrm{L}}$ with the estimated $M$ as well as $q=100 \mathrm{~nm}$ and $M_{\mathrm{L}}=1940 \mathrm{~nm}^{-1}$ (see below) to estimate $u^{*}$ using Figure 1. In the second step, $M$ and $\left\langle S^{2}\right\rangle^{1 / 2}$ were redetermined from $K c / R_{\theta}$ data at $\left\langle S^{2}\right\rangle^{1 / 2} k<u^{*}$, using eqs 2 and 3 with $P(k)=P_{\mathrm{D}}(k)$, and the process was repeated till converging $M$ and $\left\langle S^{2}\right\rangle^{1 / 2}$. Solid curves in Figure $3 b$ indicate the fitting results at the final step. Downward deviations of data points for $V_{\mathrm{e}}=8.57$ and $8.07 \mathrm{~cm}^{3}$ at small $k^{2}$ from the solid curves may come from the contamination of higher molar mass fractions due to the tailing effect of SEC. We have neglected these data points in the fitting.

\section{Molar Mass Distributions}

Figure 4 shows molar mass distributions for renatured sample S14 under the different conditions, obtained by SECMALS, where $w(M)$ is the weight fraction density of the xanthan component with the molar mass $M$. In the figure, the solid and broken curves, respectively, indicate the distribution of the native sample S14 and that after dissociation to the unimers which obtained by shifting the solid curve along the $\log M$ axis by $-\log 2$. Distributions of the renatured sample in the solutions 1 and $2\left(c_{\mathrm{r}}=0.1\right.$ or $1.0 \mathrm{mg} / \mathrm{cm}^{3}, C_{\mathrm{s}}=0.01 \mathrm{M}$; unfilled circles and triangles) are close to the broken curve, indicating that the xanthan exists almost as the dissociated unimer in the solutions, as reported by Capron et al. ${ }^{8}$ For the renatured sample in the solution $3\left(c_{\mathrm{r}}=1.0 \mathrm{mg} / \mathrm{cm}^{3}\right.$, $C_{\mathrm{s}}=0.1 \mathrm{M}$; filled circles), the distribution shifts to higher $M$ region, demonstrating that dissociated unimer chains aggregate by the addition of salt into the denatured solution. This result of $w(M)$ for the solution 3 is consistent with Kawakami et al. ${ }^{7}$ result that the average molar mass of xanthan renatured at $C_{\mathrm{s}}=0.1 \mathrm{M}$ was between those of dimer and unimer.

On the other hand, the distribution of the renatured sample in the solution $4\left(c_{\mathrm{d}}=10 \mathrm{mg} / \mathrm{cm}^{3} ; C_{\mathrm{s}}=0.01 \mathrm{M}\right)$ is close to the solid curve for the native sample. This agrees with Liu et al. ${ }^{4,5}$ result that the xanthan dimer little dissociates into two single
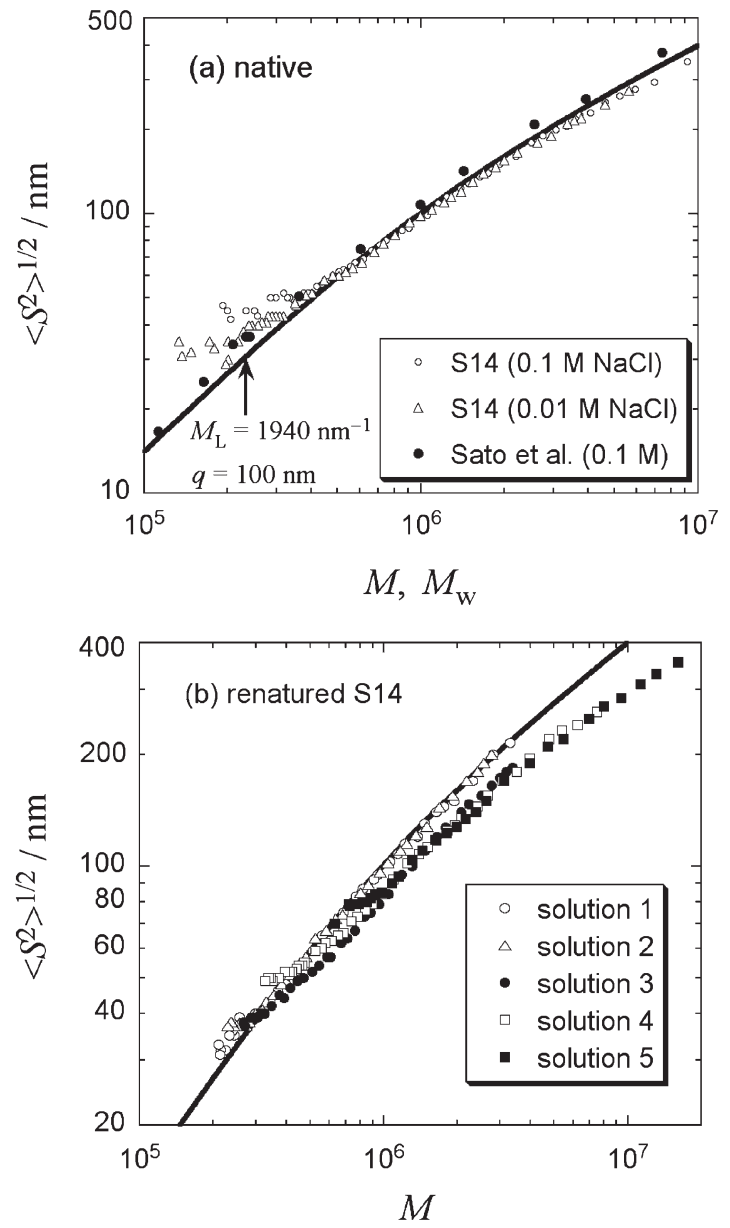

Figure 5. $M$ dependences of $\left\langle S^{2}\right\rangle^{1 / 2}$ for (a) native and (b) renatured sample of S14.

chains even at $80^{\circ} \mathrm{C}$ in $0.01 \mathrm{M}$ aqueous $\mathrm{NaCl}$. Since the aqueous xanthan solution with $c_{\mathrm{d}}=10 \mathrm{mg} / \mathrm{cm}^{3}$ contains carboxylate groups of $0.014 \mathrm{M}$ (the molar mass per repeating unit of xanthan $=920 \mathrm{~g} / \mathrm{mol}$; the degree of pyruvation of the xanthan sample $=0.3^{9}$ ), the ionic strength of the solution may be high enough to stabilize the xanthan dimer at $80^{\circ} \mathrm{C}$. In the solution $5\left(c_{\mathrm{d}}=10 \mathrm{mg} / \mathrm{cm}^{3} ; C_{\mathrm{s}}=0.1 \mathrm{M}\right)$, the distribution curve shifts to higher $M$ region than that for the native sample, demonstrating the aggregation of the denatured xanthan dimer. Although not shown, molar mass distributions of samples S1 and S5 denatured and renatured under the same condition as that of solution 5 for sample S14 also indicated the aggregation of the denatured xanthan dimer.

\section{Molar Mass Dependence of the Radius of Gyration}

Figure 5a shows $M$ dependences of $\left\langle S^{2}\right\rangle^{1 / 2}$ obtained by SEC-MALS for the native sample of S14 in $0.1 \mathrm{M}$ (unfilled circles) and $0.01 \mathrm{M}$ (triangles) $\mathrm{NaCl}$. Both data points almost agree with literature data ${ }^{4,11}$ of batch measurements for fractionated native or sonicated xanthan samples in $0.1 \mathrm{M}$ aqueous $\mathrm{NaCl}$ (filled circles) and in $0.01 \mathrm{M}$ aqueous $\mathrm{NaCl}$ (not shown). The $C_{\mathrm{s}}$ independence of $\left\langle S^{2}\right\rangle^{1 / 2}$ is due to the intrinsic high stiffness of the double helix. Slight deviations between the 
results from SEC-MALS and batch measurements may be ascribed to the difference in polydispersity of samples. While fractionated samples used previously for the batch measurements had the z- to weight-average molecular weight ratio as high as $c a .1 .2,{ }^{9}$ SEC used in this study may be more effective in the sample fractionation.

Sato et al. ${ }^{11}$ determined the persistence length $q$ and the molar mass per contour length $M_{\mathrm{L}}$ of the xanthan double helix to be $120 \mathrm{~nm}$ and $1940 \mathrm{~nm}^{-1}$, respectively, by fitting the Benoit-Doty equation ${ }^{15}$ for the wormlike chain model, given by

$$
\left\langle S^{2}\right\rangle(N)=(2 q)^{2}\left\{\frac{1}{6} N-\frac{1}{4}+\frac{1}{4 N}\left[1-\frac{1}{2 N}\left(1-\mathrm{e}^{-2 N}\right)\right]\right\}
$$

with the Kuhn statistical segment number $N\left(=M / 2 q M_{\mathrm{L}}\right)$, to their batch measurement data. On the other hand, the unfilled circles and triangles obtained by SEC-MALS are fitted to the same equation with slightly smaller $q(=100 \mathrm{~nm})$ and the same $M_{\mathrm{L}}$, as shown by the solid curve. In a low $M$ region, unfilled circles and triangles seem to deviate upward from the solid curve, which may reflect the tailing effect in the chromatogram shown in Figure 3a.

Results for the solutions 1-5 of the renatured sample S14 are shown in Figure 5b. Data points for the solutions 1 and 2 (unfilled circles and triangles) obey the solid curve fitted to the native xanthan data, which indicates the recovery of the double-helical conformation in the solutions. However, xanthan in the solutions 1 and 2 exists as the unimer, as demonstrated in Figure 4, so that the double helix must be constructed by the single chain with a hairpin loop, as proposed by Capron et al. ${ }^{8}$ Okuyama et al. ${ }^{16}$ reported the possible antiparallel arrangement of xanthan chains in the native double helix, which supports that the single xanthan chain can form the double helical structure in the anti-parallel manner.

Filled circles for the solution 3 deviate downward from the solid curve. Thus, not all denatured single xanthan chains are renatured to the double-helical unimer or dimer at $C_{\mathrm{s}}=0.1 \mathrm{M}$ but maybe to some aggregates with branched architecture. Data points for the solutions 4 and 5 (unfilled and filled squares) are also deviated downward from the solid curve, indicating that denatured xanthan dimers are not renatured to the original double helix but aggregate intermolecularly.

Figure 6 compares the molar mass $M$ dependences of $\left\langle S^{2}\right\rangle^{1 / 2}$ for aggregates formed from denatured dimers of the three xanthan samples with different $M_{\mathrm{w}}$ under the same condition of the solution 5. Not only for sample S14 but also for samples S5 and S1, the data points do not agree with the dependence expected for the native xanthan double helix (the dot-dashed curve). Therefore, even for lower molar mass xanthan samples, the original double helix are not recovered from the denatured dimers under the condition of the solution 5 .

\section{Structure of Aggregates Formed from Denatured Xanthan Dimers}

Liu et al. ${ }^{4-6}$ demonstrated that in the aqueous solution (with $c_{\mathrm{d}}>10 \mathrm{mg} / \mathrm{cm}^{3}$ ) at $80^{\circ} \mathrm{C}$ the xanthan double helix is unwound from both ends but does not dissociate into two single chains.

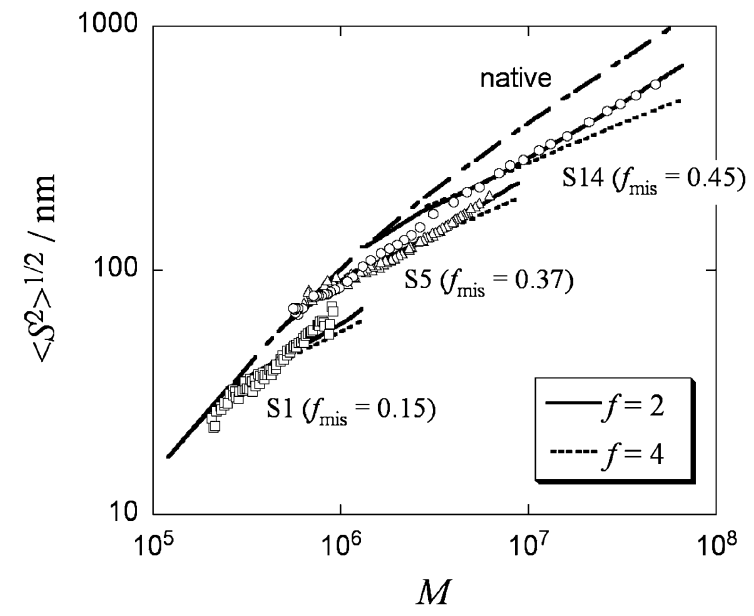

Figure 6. $M$ dependences of $\left\langle S^{2}\right\rangle^{1 / 2}$ for aggregating samples of $S 14, \mathrm{~S} 5$, and S1.

(a)

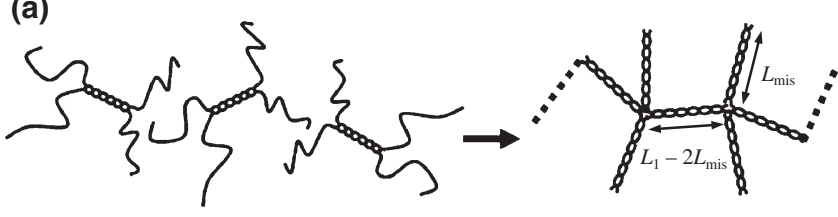

(b)

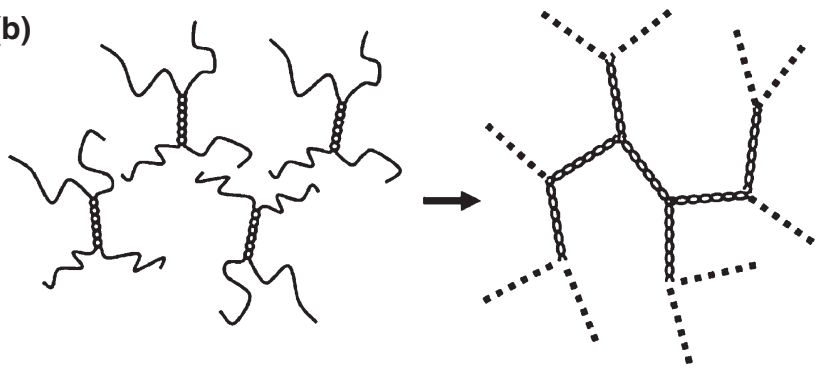

Figure 7. Schematic illustrates of (a) xanthan aggregates formed when the mismatched pairing is made by two or three partially unwound xanthan dimers and (b) aggregates formed by $m$ unwound dimers.

On the other hand, as shown in Figure 2, CD spectrum indicated that by quenching and adding the salt to the thermally denatured xanthan solution, the original double helical structure is at least locally reconstructed from the disordered random-coil conformation. Addition to this information, the results of SEC-MALS (Figure 6) showed that some portions of double helices are not reconstructed by pairing the original two chains but between different xanthan dimers to form higher aggregates.

Figure 7a schematically illustrates a linear aggregate model where each mismatched pairing between two partially unwound xanthan dimers constitutes two branches. Here, $L_{1}$ and $L_{\text {mis }}$ are the contour lengths of the native xanthan and branch of mismatched pairing, respectively. This aggregate formed by $m$ unwound dimers may be viewed as a linear chain constituted by $m+1$ monomer units of the functionality $f=2$, though monomer units at both chain ends have no branches. If the chain can freely bend at each branch point, the square radius of gyration $\left\langle S^{2}\right\rangle_{m}$ of $m$-mer may be written as ${ }^{17,18}$ 


$$
\left\langle S^{2}\right\rangle_{m}=\frac{(m+2) m}{6(m+1)} b^{2}+\left\langle S^{2}\right\rangle_{\mathrm{u}}
$$

where $b$ is the bond length of the freely jointed chain and $\left\langle S^{2}\right\rangle_{\mathrm{u}}$ is the square radius of gyration of the monomer unit. We may calculate them by

$$
b^{2}=\left\langle R^{2}\right\rangle\left(\left(1-f_{\text {mis }}\right) N_{1}\right),\left\langle S^{2}\right\rangle_{\mathrm{u}}=\left\langle S^{2}\right\rangle\left(f_{\text {mis }} N_{1}\right)
$$

where $f_{\text {mis }}$ is the mismatch fraction $\left(\equiv 2 L_{\text {mis }} / L_{1}\right), N_{1}$ is the Kuhn statistical segment number of the native xanthan sample ( $\left.\equiv L_{1} / 2 q\right)$, and $\left\langle R^{2}\right\rangle(N)$ and $\left\langle S^{2}\right\rangle(N)$ are the square end-to-end distance and square radius of gyration, respectively, for the wormlike chain with the Kuhn statistical segment number $N$. While $\left\langle S^{2}\right\rangle(N)$ is given by eq $5,\left\langle R^{2}\right\rangle(N)$ is calculated by ${ }^{12,18}$

$$
\left\langle R^{2}\right\rangle(N)=(2 q)^{2}\left[N-\frac{1}{2}\left(1-\mathrm{e}^{-2 N}\right)\right]
$$

A reviewer of this paper pointed out another aggregate model $^{23}$ for a renatured double helical polysaccharide, where each unwound xanthan dimer acts as monomer units of the functionality $f=4$, as shown in Figure 7b. For this model, the square radius of gyration may be calculated by

$$
\left\langle S^{2}\right\rangle_{m}=g_{m} \cdot \frac{m}{6} b^{2}+\left\langle S^{2}\right\rangle_{\mathrm{u}, \mathrm{H}}
$$

where $g_{m}$ is the g-factor for the polycondensate of monomer with the functionality $f=4$, and $\left\langle S^{2}\right\rangle_{\mathrm{u}, \mathrm{H}}$ is the square radius of gyration of the monomer unit of the polycondensate. According to Kajiwara, ${ }^{20}$ the g-factor for the polycondensate is given by

$$
\begin{aligned}
g_{m}= & \frac{3}{m^{2}} \frac{(m-1) !}{[(f-1) m] !} \sum_{n=0}^{m-2}(f-1)^{n} \frac{[(f-1) m-(n+1)] !}{(m-n-2) !} \\
& \times[n(f-2)+2(f-1)](n+1)
\end{aligned}
$$

On the other hand, since the monomer unit for this aggregate model can be regarded as an $\mathrm{H}$-shaped wormlike chain with a middle chain of the Kuhn segment number $N_{\mathrm{n}}=\left(1-f_{\text {mis }}\right) N_{1}$ and four end chains of the Kuhn segment number $N_{\mathrm{m}}=$ $f_{\text {mis }} N_{1} / 2,\left\langle S^{2}\right\rangle_{\mathrm{u}, \mathrm{H}}$ can be calculated from

$$
\begin{aligned}
\left(1+f_{\mathrm{mis}}\right)^{2}\left\langle S^{2}\right\rangle_{\mathrm{u}, \mathrm{H}}= & f_{\mathrm{mis}}{ }^{2}\left\langle S^{2}\right\rangle\left(N_{\mathrm{m}}\right)+\left(1-f_{\mathrm{mis}}\right)^{2}\left\langle S^{2}\right\rangle\left(N_{\mathrm{n}}\right) \\
& +f_{\mathrm{mis}}\left[\left(1+\frac{1}{2} f_{\mathrm{mis}}\right) F_{1}\left(N_{\mathrm{m}}\right)\right. \\
& \left.+\left(1-f_{\mathrm{mis}}\right) F_{1}\left(N_{\mathrm{n}}\right)+f_{\mathrm{mis}} F_{2}\left(N_{\mathrm{n}}\right)\right]
\end{aligned}
$$

with

$$
\begin{aligned}
& F_{1}\left(N_{i}\right) \equiv(2 q)^{2}\left[N_{i}-1+\left(1-\mathrm{e}^{-2 N_{i}}\right) / 2 N_{i}\right] \quad(i=\mathrm{m}, \mathrm{n}) \\
& F_{2}\left(N_{\mathrm{n}}\right) \equiv(2 q)^{2}\left[N_{\mathrm{n}}-\frac{1}{2}\left(1-\mathrm{e}^{-2 N_{\mathrm{n}}}\right)\right]
\end{aligned}
$$

(We have extended the theory of Mansfield and Stockmayer ${ }^{21}$ for $\left\langle S^{2}\right\rangle$ of the wormlike star to obtain eq 11 , by assuming the branch points to be free joints.)

Calculations of $\left\langle S^{2}\right\rangle_{m}$ for the above two aggregate models of $f=2$ (with eqs 5-8) and of $f=4$ (with eqs 9-12, and 5) need values of $N_{1}$ and $f_{\text {mis }}$. The former value may be calculated from the weight average molecular weight $M_{\mathrm{w}}$ for each sample with
$M_{\mathrm{L}}=1940 \mathrm{~nm}^{-1}$ and $q=100 \mathrm{~nm}$, by neglecting polydispersity in the molecular weight. On the other hand, we have no information about $f_{\text {mis }}$ at present, so that we treat here this as an adjustable parameter. In Figure 6, solid and dotted curves indicate theoretical values calculated for $f=2$ and 4 , respectively. For every xanthan sample, experimental data points are almost fitted to the solid curve with $f_{\text {mis }}$ indicated in the figure. The mismatch fraction $f_{\text {mis }}$ chosen increases with $M_{\mathrm{w}}$ of the sample, indicating that the probability of the mismatched pairing is higher for longer xanthan chain. The aggregate model for $f=4$ predicts weaker molar mass dependences of $\left\langle S^{2}\right\rangle^{1 / 2}$ than experiment for all the three xanthan samples, and there were no $f_{\text {mis }}$ values to give better fits to the experimental data. It may be difficult to form the mismatched pairing where two xanthan chain ends are on the opposite sides.

In Figure 6, data points for sample S14 around $M \sim 1.4 \times$ $10^{6}$ (the original $M_{\mathrm{w}}$ ) appreciably deviate downwards from the solid curve for $f=2$. The fraction in the solution 5 with $M \sim 1.4 \times 10^{6}$ may be aggregates of lower molecular weight xanthan dimers in the original sample, which were not considered in the above calculation. Since samples S1 and S5 have narrower molecular weight distributions ( $c f$. Table I), the renatured solutions of both samples shown in Figure 6 only contained fractions with $M$ higher than the original $M_{\mathrm{w}}$.

In Figure 5b, data points for the solution 3 of sample S14, which was renatured at $C_{\mathrm{s}}=0.1 \mathrm{M}$ from the dissociated single chain state, deviated downwards from the solid curve for the native xanthan double helix. This indicates that the single xanthan chain does not form the perfect double helical structure with a hairpin loop, but two single chains partially taking the double helical structure make the mismatched pairing at $C_{\mathrm{s}}=0.1 \mathrm{M}$.

Recently, Tanaka ${ }^{22}$ presented a statistical mechanical theory for the aggregation of double helical polymers in solution. In his theory, the important quantity is the association constants $\lambda_{\zeta}$ for the helical portion of the length $\zeta$ given by

$$
\lambda_{\zeta}=\sigma_{2} \exp \left(-\zeta \Delta f / k_{\mathrm{B}} T\right)
$$

where $\zeta \Delta f$ is the free energy change when a helix of length $\zeta$ is bound into a junction, $k_{\mathrm{B}} T$ is the Boltzmann constant multiplied by the absolute temperature, and $\sigma_{2}$ is the statistical weight for the initiation of a double helix.

When $\sigma_{2}=1,|\zeta \Delta f| / k_{\mathrm{B}} T \gg 1$, and the polymer concentration is dilute enough, all of the helix content $(\theta)$, the mean helix length $(\bar{\zeta} / n)$, and the number of helices per chain $(v n)$ predicted by the theory are close to unity, which means that almost perfect double helices are formed and do not aggregate so much. To increase $v n$ at keeping $\theta \approx 1$ in a dilute solution, $\sigma_{2}$ (the initiation probability of a double helix) must be increased, and under this condition $\bar{\zeta} / n$ becomes a decreasing function of the degree of polymerization $n$. This is favorably compared with the $M_{\mathrm{w}}$ dependence of $f_{\text {mis }}$ determined in Figure 6. However, since the model used by Tanaka is not identical with that used in Figure 6, we do not intend to make a quantitative comparison. 


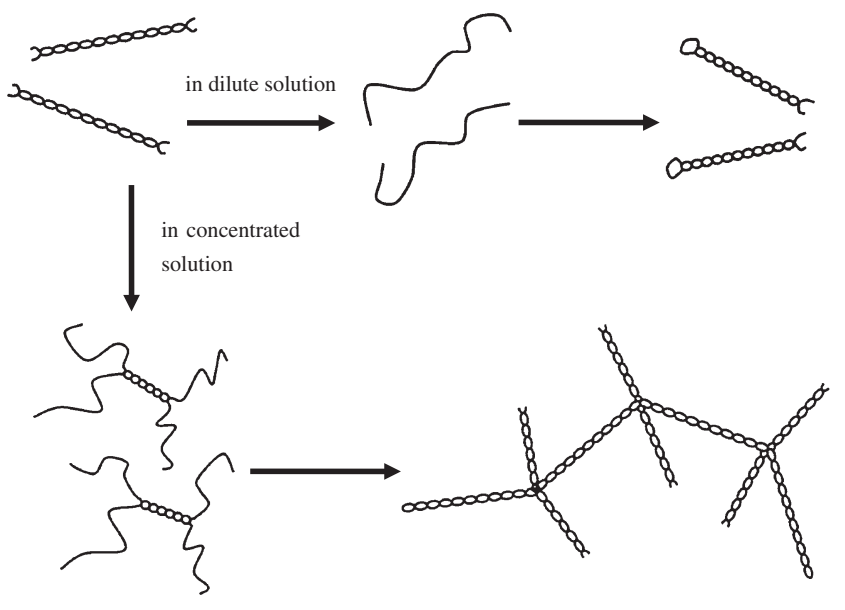

Figure 8. Schematic diagram of the denaturation and renaturation of the xanthan double helix.

\section{CONCLUSION}

We have investigated the thermal denaturation, renaturation, and aggregation behavior of the double-helical polysaccharide xanthan in aqueous solution. The behavior was sensitively dependent on the xanthan concentration and molecular weight as well as the added salt concentration. Results obtained in this study are schematically illustrated in Figure 8. In dilute solutions with $c_{\mathrm{d}} \leq 1.0 \mathrm{mg} / \mathrm{cm}^{3}$, the xanthan dimer dissociates into two single chains in $80^{\circ} \mathrm{C}$ water, and each dissociated single chain form the anti-parallel double helical structure with a hairpin loop at a sufficiently low ionic strength. (We could not check the same denaturation and renaturation behavior for the lower molecular weight samples $\mathrm{S} 1$ and S5, because the light scattering power of the two samples was too weak to make SEC-MALS measurements at $c \leq 1.0 \mathrm{mg} / \mathrm{cm}^{3}$.)

On the other hand, in solutions with $c_{\mathrm{d}}=10 \mathrm{mg} / \mathrm{cm}^{3}$, the xanthan double helix is unwound from both end portions but does not dissociate into two single chains in $80^{\circ} \mathrm{C}$ water, where xanthan itself increases the effective ionic strength to stabilize the dimer. In the renaturation process, the denatured xanthan dimer aggregates linearly by the mismatched pairing. The mismatch fraction $f_{\text {mis }}$ in the xanthan dimer $\left(\equiv 2 L_{\text {mis }} / L_{1} ; c f\right.$. Figure 7) increases with increasing the molecular weight of xanthan. The aggregation is pronounced with increasing $C_{\mathrm{s}}$.

Acknowledgment. This work was partly supported by a Grant-in-Aid for Scientific Research from the Japan Society for the Promotion of Science, as well as by a Grant-in-Aid for Scientific Research on Priority Area "Soft Matter Physics" and a Special Coordination Fund for Promoting Science and Technology (Yuragi Project) of the Ministry of Education, Culture, Sports, Science and Technology, Japan. One of the authors (Y. Matsuda) expresses his special thanks for the center of excellence (21 COE) program "Creation of Integrated EcoChemistry" of Osaka University.

Received: November 19, 2008 Accepted: March 17, 2009 Published: April 29, 2009

\section{REFERENCES}

1. T. Norisuye and A. Teramoto, in "Polymeric Materials Encyclopedia,” J. C. Salamone, Ed., CRC Press, Boca Raton, 1996, Vol. 11, p. 8801 .

2. H. W. Oviatt and D. A. Brant, Macromolecules, 27, 2402 (1994).

3. S. B. Ross-Murphy, V. J. Morris, and E. R. Morris, Faraday Symp. Chem. Soc., 18, 115 (1983).

4. W. Liu, T. Sato, T. Norisuye, and H. Fujita, Carbohydr. Res., 160, 267 (1987).

5. W. Liu and T. Norisuye, Biopolymers, 27, 1641 (1988).

6. W. Liu and T. Norisuye, Int. J. Biol. Macromol., 10, 44 (1988).

7. K. Kawakami, Y. Okabe, and T. Norisuye, Carbohydr. Polym., 14, 189 (1991).

8. I. Capron, G. Brigand, and G. Muller, Polymer, 38, 5289 (1997).

9. T. Sato, T. Norisuye, and H. Fujita, Polym. J., 16, 341 (1984).

10. Y. Takada, T. Sato, and A. Teramoto, Macromolecules, 24, 6215 (1991).

11. T. Sato, T. Norisuye, and H. Fujita, Macromolecules, 17, 2696 (1984).

12. H. Yamakawa, "Modern Theory of Polymer Solutions," Harper \& Row, New York, 1971.

13. T. Yoshizaki and H. Yamakawa, Macromolecules, 13, 1518 (1980).

14. E. R. Morris, D. A. Rees, G. Young, M. D. Walkinshaw, and A. Darke, J. Mol. Biol., 110, 1 (1977).

15. H. Benoit and P. Doty, J. Phys. Chem., 57, 958 (1953).

16. K. Okuyama, S. Arnott, R. Moorhouse, M. D. Walkinshaw, E. D. T. Atkins, and C. Wolf-Ullish, in "Fiber Diffraction Methods," A. D. French and K. H. Gardner, Ed., American Chemical Society, Washington, DC, 1980, p. 411.

17. P. J. Flory, "Statistical Mechanics of Chain Molecules," Interscience, New York, 1969.

18. H. Yamakawa, "Helical Wormlike Chains in Polymer Solutions," Springer-Verlag, Berlin \& Heidelberg, 1997.

19. T. Sato and Y. Matsuda, Polym. J., 41, 241 (2009).

20. K. Kajiwara, Polymer, 12, 57 (1971).

21. M. L. Mansfield and W. H. Stockmayer, Macromolecules, 13, 1713 (1980).

22. F. Tanaka, Macromolecules, 36, 5392 (2003).

23. L. Piculell, in "Food Polysaccharides and their Applications," A. M. Stephen, Ed., Marcel Dekker, 1995, pp 205-244. 